\title{
A guideline for the prevention and management of Fetal Alcohol Spectrum Disorder in South Africa
}

\author{
Babatope O. Adebiyi ${ }^{1 *}$ (D) Ferdinand C. Mukumbang ${ }^{1}$ (D) and Anna-Marie Beytell ${ }^{2}$ (D)
}

\begin{abstract}
Background: Fetal Alcohol Spectrum Disorder (FASD) is a public health problem globally, with South Africa having the highest recorded prevalence of all countries. Government programmes to prevent and manage FASD remain limited because of the lack of a specific policy. Herein, we developed a guideline to inform policy on the prevention and management of FASD in South Africa.
\end{abstract}

Methods: We applied a modified version of the World Health Organization's approach to guideline development in three phases. In the first phase, we designed the initial guideline prototype. To do this, we conducted an in-depth interview with policymakers and a focus group with relevant service providers on policy requirements for FASD, a document review of policies on FASD and a scoping review of various interventions for FASD. In phase 2, we refined the initially formulated guideline prototype through a discursive approach with seven local and international experts on FASD. Phase 3 involved refining the prototype using a modified Delphi approach. Fortythree and forty-one experts participated in rounds 1 and 2 of the Delphi approach, respectively. The acceptable consensus for each included policy statement was $85 \%$.

Results: We identified three aspects of the proposed guideline, which are the approaches and guiding principles, the prevention measures and the management measures. The guideline proposes that a FASD policy should consider lifespan needs, be culturally diverse, collaborative, evidence-based, multi-sectoral and address social determinants of health contributing to FASD. The essential components of FASD prevention policy consist of awareness and education of the dangers of drinking alcohol, access to treatment for alcohol problems and training of service providers. The management components include capacity building related to diagnosis, educating parents regarding the needs and management, appropriate referral pathways, training of teachers regarding classroom management and support for parents and individuals with FASD.

Conclusion: FASD in South Africa deserves urgent attention. Developing a specific policy to guide programmes could enhance and coordinate the efforts towards preventing and managing FASD. The guideline has the potential to assist policymakers in the development of a comprehensive and multi-sectoral policy for prevention and management of FASD, considering the consensus obtained from the experts.

Keywords: Fetal alcohol spectrum disorder, Policy, Guideline, Delphi approach, Management, Prevention, Snowball sampling, Developmental disabilities, Women

\footnotetext{
* Correspondence: atommega@yahoo.com

'School of Public Health, University of the Western Cape, Cape Town 8001,

South Africa

Full list of author information is available at the end of the article
}

(c) The Author(s). 2019 Open Access This article is distributed under the terms of the Creative Commons Attribution 4.0 International License (http://creativecommons.org/licenses/by/4.0/), which permits unrestricted use, distribution, and reproduction in any medium, provided you give appropriate credit to the original author(s) and the source, provide a link to the Creative Commons license, and indicate if changes were made. The Creative Commons Public Domain Dedication waiver (http://creativecommons.org/publicdomain/zero/1.0/) applies to the data made available in this article, unless otherwise stated. 


\section{Contributions to the literature}

- How to adapt WHO's approach for guideline development to develop a guideline for policy.

- How to develop/improve a guideline/policy using a multi-method study with a modified Delphi approach.

- Policymakers', service providers' and experts' perspectives regarding policy requirements for the prevention and management of FASD.

- The essential components of FASD policy, which include awareness and education of the dangers of drinking alcohol, access to treatment for alcohol problems, training of service providers, capacity building related to diagnosis and support for parents and individuals with FASD.

\section{Background}

Globally, one in every 13 alcohol-exposed pregnancies results in a Fetal alcohol spectrum disorder (FASD) [1]. In 2017, the global prevalence of FASD was 8 per 1000 children and youth [1]. In 2016, the Foundation for Alcohol Related Research (FARR), estimated that 6 million individuals were affected by FASD in South Africa [2] ranging from 29 to 290 per 1000 live births, the highest recorded prevalence in any part of the world [3]. The prevalence of FASD varies from one province to another, with the Western Cape and Northern Cape provinces the most affected. In 2017, the prevalence of FASD among Grade 1 pupils in the Western Cape was estimated to be 196 to 276 per 1000 [4], while in the Northern Cape the prevalence was estimated at 63.9 per 1000 Grade 1 pupils in 2015 [5].

Although FASD can be clinically identified through characteristic facial features that can be observed on examination [6, 7], some FASD are considered as hidden disabilities because in most cases there are no noticeable physical manifestations [8-11] thus increasing the chances of missed diagnosis or misdiagnosis [12-15]. Misdiagnosis and missed diagnoses prevent individuals from accessing early and appropriate services [16]. To diagnose any of the FASD (fetal alcohol syndrome $\{F A S\}$, partial FAS, $\{$ PFAS\}, alcohol-related neurodevelopmental disorder \{ARND\} and alcohol-related birth defects $\{A R B D\})$, individuals must meet all or some of the range of identified criteria [17-19]. These criteria include documented or undocumented prenatal alcohol exposure, prenatal and or postnatal growth deficiency, deficient brain growth and neurobehavioral impairment - with or without cognitive impairment [17-19]. The requirements for prevention, diagnosis and management suggests a multi-sectoral approach [3, 9, 20-23].

While the South African government endeavours to address the high prevalence of FASD, its responses through policy have not been adequate [24, 25]. Despite the availability of context-relevant evidence-based interventions such as case management and universal prevention approaches to prevent FASD [26, 27], there is a lack of policy and resources to guide the expansion and implementation of these interventions to many parts of the country. There is evidence that current prevention and management interventions are informed by geneticrelated and other generic policy documents [21, 28]. For instance, the Human Genetics Policy Guidelines for the Management and Prevention of Genetic Disorders, Birth Defects and Disabilities and the Mini Drug Master Plan covers FASD as a genetic disease. The National Department of Health also recognises FASD as one of the ten focal genetic conditions. The Western Cape government has listed FASD as a provincial health priority in its services regarding birth defects [29]. Because these documents reflect FASD in a generic manner, they do not support the holistic and comprehensive (multi-sectoral) approach required to address FASD [24, 25, 30].

In addition to the efforts of the South African national and provincial governments, non-profit organisations (NPO) provide various levels of contributions to address FASD in South Africa. The South African government provides partial funding to some of these organisations. These organisations function mainly in the Western Cape, though they have a presence in other provinces, especially Northern Cape and Eastern Cape. FARR, for example, provides services such as mentorship, creating social awareness, providing education and training programmes, conducting medical and psycho-social FASDrelated research and providing support and diagnostic services [31]. FASfacts, as an NPO, offers FASD prevention programmes through experiential learning, advertising campaigns using churches, films and theatres to pass FASD messages and mentoring of pregnant women [32]. These organisations obtain funding from the government by aligning their programmes to the existing generic policies.

Different approaches have been proposed to develop relevant and effective FASD policies. One such approach proposed to prevent and manage FASD, is the decolonisation policy discourse [33]. The proponents of the decolonised policy discourse advocate that women should be seen as victims of the FASD problem, not as perpetrators [33]. They also propose that the socio-economic and sociopolitical circumstances that predispose women to alcohol consumption during pregnancy, which may lead to FASD, should be addressed [33]. Other proposed approaches are the comprehensive (addressing all factors); inclusive (involving all sectors and all levels of the government); and the human rights-based approach (acknowledging the principles of non-discrimination, participation, inclusion, equity and access) [34]. A women-centred approach, which 
considers the needs of women in all aspects of design and delivery, including the location and accessibility of services, staffing, programme development, content and materials has also been proposed [34]. We propose that for an FASD policy to be truly comprehensive and multi-sectoral, all the above approaches should be scrutinised.

Having a multi-sectoral and comprehensive policy [21,35] as demonstrated by the Canadian and Australian governments [34, 36, 37], has the potential to coordinate existing and new approaches and programmes for preventing and managing FASD. In South Africa, government programmes to address FASD remain limited partly because of the absence of a policy to inform prevention and management [29]. To this end, we sought to develop a guideline to inform the design of a comprehensive and multi-sectoral policy to address FASD in South Africa [30].

\section{Methods}

In developing the guideline for the prevention and management of FASD, we adapted the World Health Organization's approach (steps) [38, 39], which we organised in three phases (Fig. 1). The first phase entailed designing the initial guideline prototype. In Phase 2, we refined the initially formulated guideline prototype. Phase 3 involved testing and confirming the refined prototype using a modified Delphi approach.

\section{Phase 1: designing the guideline prototype}

We conducted focus group discussions with service providers and in-depth interviews with policymakers to identify policy requirements for FASD in South Africa $[25,30]$. The service providers included educators, health and allied health professionals as well as social service professionals providing services to women, especially pregnant women or individuals with FASD or related conditions. The policymakers included individuals working in the Departments of Social Development, Health and Education on FASD-relevant issues or related conditions. The service providers and the policymakers were asked various questions regarding current practices and interventions and policy requirements for FASD.

In addition to the focus group discussions and in-depth interviews, we conducted a document review. We identified clauses of FASD policy in various South Africanrelated policy documents [28]. We searched databases (PubMed and Google search engines) and the websites of South African national and provincial departments. We used the following search terms: foetal alcohol spectrum disorder, alcohol-related neurodevelopmental disorder, foetal alcohol syndrome, white paper, green paper, policy, action plan, gazette and South Africa. Although, we used "foetal" for the search, it includes documents with "fetal". We also contacted the designated persons in the departments of Education, Health, Social Development and Trade and Industry via emails to request other relevant documents.

Furthermore, we conducted a scoping review to identify the prevention and management interventions of FASD globally that could be included in the policy [38]

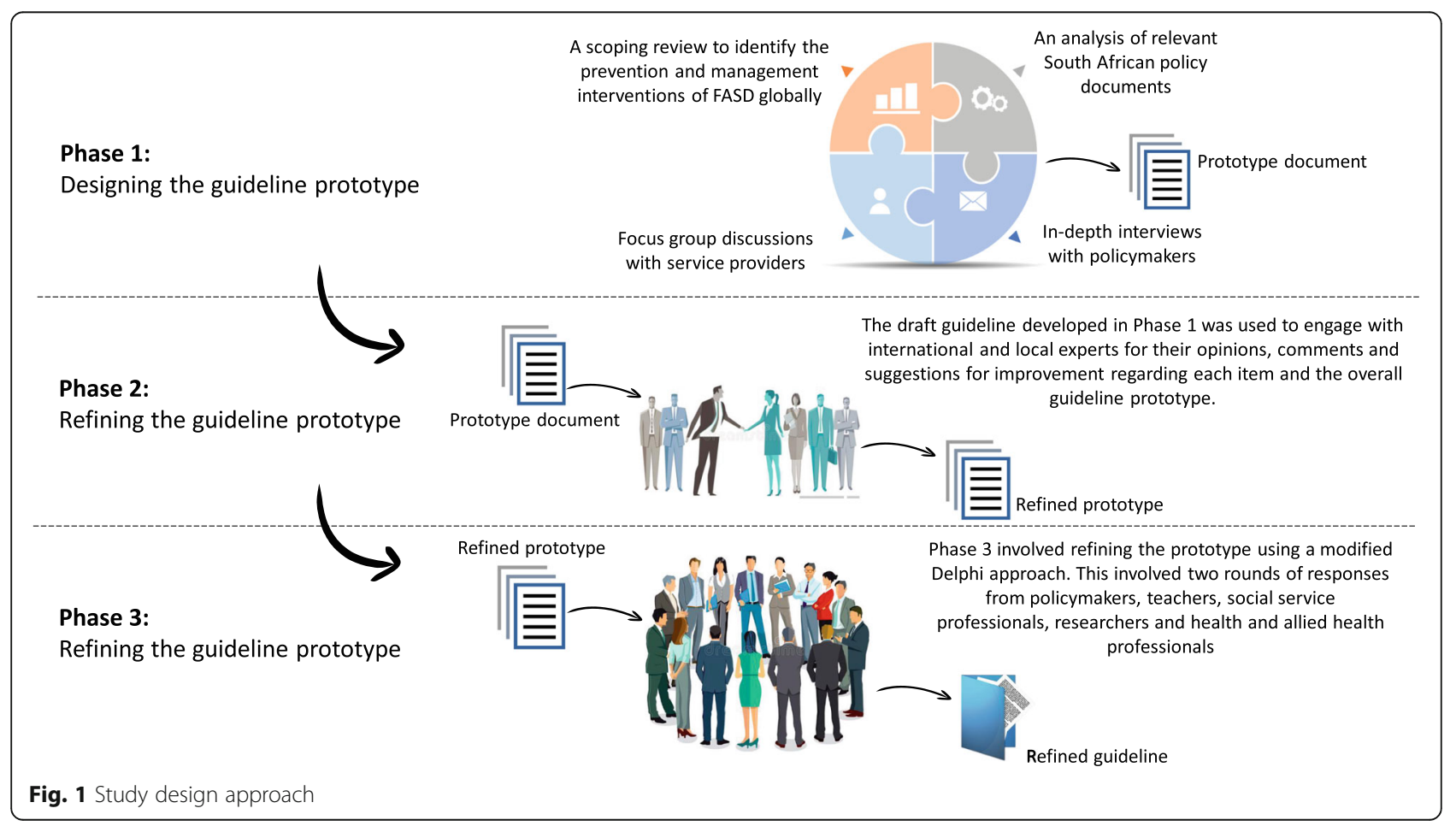


We searched the following Ebsco Host embedded databases: Academic Search Complete, ERIC, SoINDEX, Health Source. We also searched the Nursing/Academic Edition, CINAHL, Medline and Psych-ARTICLES, Springer Links, SAGE Journals and PubMed databases. The search terms used include fetal alcohol spectrum disorder, fetal alcohol syndrome, alcohol-related neurodevelopmental disorder, alcohol-related birth defects, partial fetal alcohol syndrome, prenatal alcohol exposure, intervention, strategy, treatment, programme, management, prevention and therapy.

Data obtained from the various sources were analysed using content and thematic framework analyses in the different studies. The findings from these studies were integrated towards developing the initial guideline prototype using the framework indicated in Fig. 2.

The information gleaned from the various sections as represented in the framework were then rephrased into different statements to formulate a draft guideline.

\section{Phase 2: refining the initial guideline prototype}

The draft guideline developed in Phase 1 was used to engage with seven international and local experts (Table 1) for their opinions, comments and suggestions for improvement regarding each item and the overall guideline prototype. These experts were purposely sampled based on their availability and expertise. Emails were sent to them requesting their participation. We selected those who had experience in conducting FASD research and those who had been involved in developing FASD policies.

Following the engagement with the experts, we obtained a refined guideline prototype (Table 2).

\section{Phase 3: testing and finalising the guideline}

We conducted a modified Delphi approach to improve the prototype guideline developed for policy on FASD.

\section{Development of questionnaire}

The statements in the refined prototype developed in Phase 2 (Table 2) were used to design Likert scale statements for the Delphi responses. We asked the respondents to rate

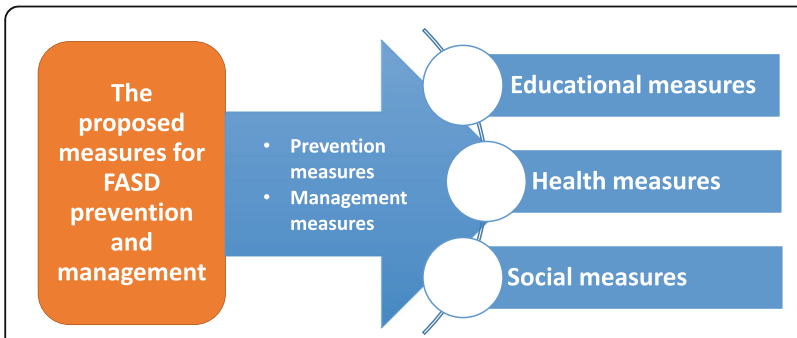

Fig. 2 Heuristic classification framework applied to summarise the data
Table 1 Characteristics of international and local experts

\begin{tabular}{lll}
\hline Type of expert & Sex & Position \\
\hline International & Female & Professor \\
International & Female & Researcher \\
International & Female & Policymaker \\
International & Male & Researcher \\
Local & Male & Professor \\
Local & Female & Researcher \\
Local & female & Lecturer \\
\hline
\end{tabular}

their agreement with each statement on a 5-point Likert scale (from 'strongly disagree' to 'strongly agree') whereby they could answer 'neutral' when they were not sure how to rate a statement. The questionnaire was divided into three sections (principles and approaches to policy development, prevention considerations and management consideration) and three sub-sections (education, health and social aspects). The participants were encouraged to provide further comments at the end of each section and sub-section. The questionnaire was piloted among international and local experts working on the prevention and management of FASD to ensure coherence, feasibility and validity.

\section{Recruitment of the participants}

Using purposive and snowball sampling techniques, we recruited professionals who have experience and or expertise on FASD. First, we purposively recruited 15 participants (policymakers, teachers, social service professionals, researchers and allied health professionals) as the seed participants. We used the following criteria for selecting the first batch of participants (i) having experience working with women (especially pregnant women) and or individuals with FASD or related conditions, (ii) experience in making or implementing FASD policy or related conditions, and (iii) having published articles or conducted FASD research or related conditions. Second, we asked the 15 participants to invite others in their networks (snowballing). Through purposive and snowball approaches, 43 participants completed round 1 of the Delphi study. We invited the 43 respondents who participated in round 1, and 41 completed the round 2 questionnaire. In Table 3, the characteristics of the participants in both rounds of the Delphi study are shown.

\section{Data collection}

We created a Google form, which allowed the participants to respond online and invited them via email to follow the link to this form. Participants were given an initial period of 2 weeks to respond to the questionnaire. The 2 weeks were extended to 2 months to increase participation including the participants recruited by snowballing. We reminded those who had not responded 
Table 2 The prototype guideline for the prevention and management of the FASD policy

Approaches and guiding principles and approaches - the proposed FASD
policy should be...
Holistic
User- and caregiver-focused
Inter-departmental/multi-sectoral
Considerate of needs across the lifespan
Collaborative
Human rights-based
Based on a public health framework
Culturally diverse and culturally sensitive
Evidence-based
Woman/family-centred
Clear about referral pathways
Designed to avoid victim blaming
Cost-effective
Driven by behavioural economics

Education-related proposed prevention measure for FASD - the proposed FASD policy should...

Enhance awareness of the dangers of drinking alcoholic beverages during pregnancy in schools including colleges and universities

Assist individuals with alcohol-use problems in educational settings to access treatment

Address barriers to access treatment for alcohol-related problems in educational settings

Address stigma associated with alcohol abuse in educational settings

Facilitate training of teachers re the FASD prevention/ awareness programme

Facilitate the development and implementation of FASD awareness programmes in schools (including colleges and universities)

Facilitate the use of peer education for the FASD awareness programme in schools

Promote a healthy lifestyle in schools through sport and other extra-curricular activities

Make school events alcohol-free

Discourage the establishment of liquor stores in the proximity of schools

Promote the education of young individuals about healthy pregnancy in schools

Health-related proposed prevention measures for FASD - should...

Facilitate screening for alcohol use in clinics and hospitals

Encourage proper documentation of the alcohol history of women, especially pregnant women

Facilitate the inclusion of FASD prevention as a part of health promotion activities in clinics and hospitals

Facilitate the education of individuals and couples re the dangers of drinking alcoholic beverages during pregnancy in the pre-conception clinic

Facilitate the education of individuals and couples re the dangers of drinking alcoholic beverages during pregnancy in the reproductive clinic

Encourage the use of visible posters and pamphlets for FASD
Table 2 The prototype guideline for the prevention and management of the FASD policy (Continued)

prevention campaigns in all clinics and hospitals

Facilitate training of healthcare professionals re FASD prevention

Facilitate early and appropriate referral to treatment for individuals (including women) with alcohol misuse issues

Empower health professionals with the skills to counsel and ask questions about safe and appropriate alcohol use

Promote the use of contraceptives to avoid unplanned pregnancy

Community/social-related proposed prevention measures for FASD - should...

Facilitate public awareness re the dangers of alcohol abuse

Facilitate the education of all people in the community re the dangers of drinking alcohol during pregnancy

Facilitate the education of individuals and couples re the dangers of drinking alcohol during pregnancy

Encourage the use of community groups for FASD prevention (education and awareness)

Facilitate the training of the community health/community-based workers and youth care/social workers re FASD prevention

Facilitate early intervention and assistance for individuals with alcoholuse problems in the community

Facilitate the creation of social programmes such as skills training and empowerment programmes for women in the community

Encourage awareness and education re FASD in the workplace, rural and urban areas and farming communities

Promote the use of multimedia such as posters, adverts, pamphlets, TV, social media and road shows re FASD awareness in the communities

Promote enforcement of liquor laws and regulation of shebeens to control accessibility and availability of alcohol in the community

Provide access to treatment for people with alcohol-use problems in the community

Provide smooth aftercare and community reintegration to people who have attended alcohol rehab

Promote afterschool activities in the community to prevent early exposure of adolescents to alcohol

Discourage all advertisements that link alcohol to sport/other popular community events/activities

Mandate labels on alcohol containers to contain information re the dangers of drinking alcoholic beverages during pregnancy

Mandate that liquor stores display warning signs regarding alcohol and pregnancy

Enable the creation of support groups for individuals with alcohol misuse issues in the community

Facilitate the training of the community and religious leaders re FASD prevention

Promote collaboration and the use of non-profit organisations (NPO) re FASD prevention

Utilise community and religious leaders to increase FASD awareness among their communities

Promote the expansion and adoption of NPO evidence-based interventions re prevention in the community

Assist families to support individuals with alcohol-use problems

Education-related proposed management measures for FASD - should...

Facilitate the development of a curriculum that accommodates individuals with FASD 
Table 2 The prototype guideline for the prevention and management of the FASD policy (Continued)

\footnotetext{
Facilitate training of teachers re the classroom management for individuals with FASD

Promote skilled schools for learners with learning disabilities (including individuals with FASD) who are not benefiting from formal education

Make provision for special assistance for individuals with FASD within mainstream schools

Facilitate the creation of the special schools for learners with a learning disability (including individuals with FASD) who are not benefiting from mainstream schooling

Facilitate the education of parents re the needs and management of individuals with FASD

Health-related proposed management measures for FASD - should...

Facilitate capacity building re diagnosis among health professionals

Facilitate FASD screening for all children who are known to have been prenatally exposed to alcohol

Make provision for diagnostic services for individuals

Promote diagnosis for school children, adolescents and adults to reduce rates of people who are left undiagnosed or misdiagnosed

Promote appropriate referral pathways to services after diagnosis

Facilitate the creation of diagnostic centres in clinics, hospitals and communities
}

Facilitate the creation of national surveillance for FASD via reports from health professionals

Make provision for integrated and individualised medical services for individuals with FASD

Encourage routine consideration of FASD in the diagnosis and management of mental illness and developmental disorders

Community/social-related proposed management measures for FASD - should.

Provide skills training and empowerment programmes for those in need among individuals with FASD

Facilitate appropriate employment opportunities for individuals with FASD

Facilitate the training of community health workers/community-based workers/youth care workers/social workers and professionals within judiciary system re FASD management

Facilitate the training of the biological and foster parents/caregivers regarding the management of FASD

Promote the empowerment of the parents/caregivers of individuals with FASD in the community

Promote the establishment of support systems for biological and foster parents/caregivers and individuals with FASD in the community

Promote the referral of parents and individuals with FASD to appropriate services

Make provision for effective counselling services for parents and individuals with FASD

Encourage family/community support for individuals with FASD

Provide support for individuals with FASD in child protection/foster care and the criminal justice system

Facilitate the creation of a structure and supportive environment at home, school and beyond
Table 3 Characteristics of the participants

\begin{tabular}{lll}
\hline Characteristics & Round $1(n=43)$ & Round 2 $(n=41)$ \\
\hline Gender & 11 & 11 \\
Male & 32 & 30 \\
Female & & \\
Occupation & 14 & 13 \\
Social services provider & 9 & 9 \\
Researcher & 12 & 11 \\
Policymaker & 5 & 5 \\
Allied health and health & 3 & 3 \\
Teacher & & 1 \\
Highest level of education & 1 & 3 \\
High school & 3 & 37 \\
College & 39 & 13 \\
University & & 11 \\
Years of experience & 14 & 7 \\
1-5 & 12 & 10 \\
6-10 & 7 & 10
\end{tabular}

before the end of the 2 weeks as well as weekly up to the 2 months. The round 1 questionnaire allowed participants not only to agree or disagree with a set of statements but also to provide comments to improve the guideline. Before we administered the questionnaire, we agreed that if a statement reached at least $85 \%$ consensus from the participants, it would be endorsed. Statements not reaching the $85 \%$ consensus, and the new ones generated from the analysis of the comments of the participants in round 1 , were prepared as a separate questionnaire and administered in round 2.

\section{Validity}

To ensure validity in this study, we consulted with local and international experts to ensure the content covered all the areas of the variable being measured. The experts' opinions were used to improve the guideline before it was used for the Delphi study. We also consulted questionnaires and surveys designed to measure similar concepts.

\section{Reliability}

To ensure reliability, we asked some of the participants to answer the questionnaire for a second time. Both their responses were compared to show consistency. Additionally, we calculated Cronbach's alpha for the study using the Statistical Package for Social Science (SPSS) software. The Cronbach's alpha for round 1 is 0.977 , and round 2 is 0.796 . 


\section{Data analysis}

We analysed the quantitative data using SPSS, generating descriptive statistics (frequencies) for each statement.

\section{Ethical considerations}

The research ethics committee of the University of the Western Cape approved this study (BM/16/4/4). Approvals were also obtained from the Western Cape Department of Education (20161212-6937), Departments of Health (WC 2016RP29_862) and Social Development (12/1/2/4). Eligible experts were invited to participate via email with the inclusion of an information sheet and a consent form. The experts were required to read the information sheet to understand the purpose of the study and what they needed to do if they agreed to participate. Those who agreed to participate were asked to sign the consent form. We maintained confidentiality during the research by anonymising the participants.

\section{Results}

\section{Guiding principle and approach}

Three of the statements achieved 100\% consensus in round 1 and none in round 2 (Additional file 1a). Women/family-centeredness attained $84 \%$ consensus in round 1 and based on the comments made by some of the participants, we decided to separate family from women in round 2. The participants said having women-centred as one of the approaches and guiding principle could promote stigma and blame games. In round 2, family-centredness reached 95\% consensus while women-centredness only achieved $68 \%$ agreement. However, the policy should be designed to avoid genderfocused interventions recorded the lowest consensus of $66 \%$. There was no particular reason given by the participants for the latter.

\section{Proposed prevention measure}

\section{Education-related proposed prevention measure}

In round 1, only one of the statements attained $100 \%$ agreement from the respondents with none in round 2 . However, most statements achieved $90 \%$ and greater in terms of agreement. The statement, 'Make school events alcohol-free' attained the lowest agreement at $81 \%$. When administered in round 2 , it only obtained an agreement rate of $78 \%$ (Additional file $1 \mathrm{~b}$ ).

\section{Health-related proposed prevention measures}

Five of the statements on health-related proposed prevention measures reached $100 \%$ consensus among participants in round 1 (Additional file 1c). The only statement generated from the comments made by participants in round 1 and administered in round 2 attained $93 \%$ agreement.
Community/social-related proposed prevention measures

Eight statements had $100 \%$ consensus in round 1 while one attained $100 \%$ agreement in round 2 . The remaining statements achieved agreement in $90 \%$ and above except two (statements 14 and 23). 'Discourage all advertisements that link alcohol to sport/other popular community events/activities' was re-administered in round 2 and recorded a lower consensus (71\%) than round 1 . Some of the participants commented that a ban on the advertisement has little or no impact on reducing or preventing alcohol consumption during pregnancy in an area where drinking is rampant (Additional file 1d).

\section{Proposed management measures}

\section{Education-related proposed management measures}

Of the six education-related proposed measures, only one attained $100 \%$ of agreement in round 1 . The remaining statements also achieved a consensus rate of $90 \%$ and above (Additional file 1e). Therefore, none of the statements was administered in round 2 .

\section{Health-related proposed management measures}

None of the health-related proposed management measures achieved $100 \%$ agreement in both rounds (Additional file 1f). Five of the statements reached consensus at $90 \%$ and above. The remaining statements attained $85 \%$ or more consensus, except one. Although all the statements in this sub-section reached the acceptable consensus for this study, some of the participants raised concerns. They commented on the feasibility and practicality of diagnosing school children, adolescents and adults, individualised medical services and creating national surveillance. They cautioned that it is not advisable to diagnose without making provision for services after diagnosis. They reported that individualised services would be too expensive and appropriate logistics have to be implemented for national surveillance. In round 2 , we decided to separate integrated and individualised medical services. Only integrated service reached the consensus (88\%) acceptable in this study.

\section{Community/social-related proposed management measures} None of the statements attained $100 \%$ consensus in round 1, whereas one reached $100 \%$ agreement in round 2 (Additional file 1g). Besides one statement, all the others achieved a consensus of $90 \%$ or more. The promotion of grant/social welfare for individuals with FASD attained the lowest consensus (49\%). One of the reasons given for the low consensus was that promoting grants and social welfare would encourage women to drink during pregnancy to receive these benefits.

Following the two rounds of responses in the Delphi process, we selected only those statements that qualified by $85 \%$ in either round to be included in the guideline (Table 4). 
Table 4 A proposed guideline for policy on the prevention and management of FASD.

Overall guiding principles of the policy

The panel agreed that policy to inform the prevention and management of FASD should

- Be holistic, considering the prevention, diagnosing and management of FASD.

- Consider the individuals with FASD and their caregivers.

- Involve all relevant government departments such as the departments of health, education, justice, social development, trade and industry, labour.

- Consider the needs of individuals with FASD throughout their lifespan.

- Involve the collaborative action of various professionals (social service, justice and healthcare); healthcare professionals from the doctors, midwives, nurses, to the community healthcare workers.

- Be holistic, considering the prevention, diagnosing and management of FASD.

- Adopt a human rights-based approach, which protects and promotes the rights of women, children, families and communities affected by FASD and recognises the principles of non-discrimination, participation, inclusion, equity and access.

- Adopt a public health framework, which acknowledges drinking during pregnancy and FASD are part of a complex interplay of biological, social, psychological, environmental and economic factors.

- Be culturally diverse and culturally sensitive, acknowledging the importance and strength of cultural values and norms.

- Use relevant and current evidence to inform practice and interventions to strengthen the knowledge base to effectively prevent and manage FASD.

- Establish clear referral pathways for the effectiveness of the prevention and management FASD

- Avoid victim blaming that is placing women at the centre of the FASD problem, which will not consider them as the perpetrators of the problem.

- Consider cost-effective interventions, which encourages a populationwide approach and enhances a wider coverage.

- Be family-centred, service providers must acknowledge and value the need for individuals within the family structure.

- Address social determinants of health contributing to FASD.

- Consider input from individuals with FASD and their families in developing a policy for the prevention and management of FASD.

- Promote responsible parenting, which recognises human values and enhance the development of individuals with FASD.

FASD prevention measures

Education-related prevention measures for FASD

The panel agreed that an FASD policy on education-related prevention should contain strategies to

- Increase awareness of the dangers of drinking alcoholic beverages during pregnancy in educational settings.

- Assist individuals with alcohol-use problems in educational settings to access treatment.

- Address the barriers to access treatment for alcohol-related problems in educational settings.

- Address stigma associated with alcohol abuse in educational settings.

- Improve training of teachers on FASD prevention/awareness programme.

- Facilitate the development and implementation of FASD awareness
Table 4 A proposed guideline for policy on the prevention and management of FASD. (Continued)

programmes in educational settings.

- Facilitate the use of peer education for FASD awareness programme in educational settings.

- Promote healthy lifestyle in schools through sport and other extracurricular activities.

- Discourage the establishment of the liquor stores in the proximity of educational settings.

- Promote the education of young individuals about healthy pregnancy in educational settings.

- Facilitate the teaching of responsible parenthood in educational settings.

- Improve the teaching of safe sex practices in educational settings.

Health-related prevention measures for FASD

The panel agreed that an FASD policy on health-related prevention should contain strategies to

- Improve the screening of alcohol use in clinics and hospitals.

- Improve documentation on the alcohol history for women, especially pregnant women.

- Facilitate the inclusion of FASD prevention as a part of health promotion activities in clinics and hospitals.

- Improve the education of individuals and couples on the dangers of drinking alcoholic beverages during pregnancy in the pre-conception clinic.

- Improve the education of individuals and couples on the dangers of drinking alcoholic beverages during pregnancy in the reproductive clinic.

- Encourage the use of visible posters and pamphlets for FASD prevention campaigns in all clinics and hospitals.

- Improve the training of healthcare professionals on FASD prevention.

- Improve early and appropriate referral to treatment for individuals (including women) with alcohol misuse issues.

- Empower health professionals with the skills to counsel and ask questions about alcohol use in a safe and appropriate way.

- Promote the use of contraceptives to avoid unplanned pregnancy.

- Improve assistance to parents of individuals with FASD to avoid having another child with FASD.

Community/social-related prevention measures for FASD

The panel agreed that an FASD policy on community-related prevention should contain strategies to

- Improve education and public awareness of the dangers of alcohol abuse.

- Improve the education of all people in the community on the dangers of drinking alcohol during pregnancy.

- Improve the education of individuals and couples on the dangers of drinking alcohol during pregnancy in the community.

- Encourage the use of community groups for FASD prevention (education and awareness).

- Improve the training of the community health /community-based workers and youth care/social workers on FASD prevention.

- Facilitate early intervention and assistance for individuals with alcoholuse problems in the community.

- Facilitate the creation of social programmes such as skills training and empowerment programmes for women in the community.

- Improve awareness and education on FASD in the workplace, rural and urban areas and farming communities.

- Promote the use of multimedia such as posters, adverts, pamphlets, TV, 
Table 4 A proposed guideline for policy on the prevention and management of FASD. (Continued)

social media and road shows for FASD awareness in the communities.

- Improve enforcement of liquor laws and regulation of shebeens to control accessibility and availability of alcohol in the community.

- Improve access to treatment for people with alcohol use problems in the community.

- Improve smooth aftercare and community reintegration for people who have attended alcohol rehab.

- Promote afterschool activities in the community to prevent early exposure of adolescents to alcohol.

- Mandate labels on alcohol containers to contain information on the dangers of drinking alcoholic beverages during pregnancy.

- Mandate that liquor stores have warning signs regarding alcohol and pregnancy.

- Enable the creation of support groups for individuals with alcohol misuse issues in the community.

- Facilitate the training of the community and religious leaders on FASD prevention.

- Promote collaboration and the use of non-profit organisation (NPO) for FASD prevention.

- Utilise the community and religious leaders to increase FASD awareness among their communities.

- Promote the expansion and adoption of NPO evidence-based interventions for prevention in the community.

- Improve assistance to families to support individuals with alcohol use problems.

- Improve interventions services for mothers who have a child with FASD in the community.

FASD management measures

Education-related management measures for FASD

The panel agreed that an FASD policy on education-related management should contain strategies to

- Facilitate the development of a curriculum that accommodates individuals with FASD.

- Improve the training of teachers on classroom management for individuals with FASD.

- Promote skill schools for learners with learning disabilities (including individuals with FASD) that are not benefiting from formal education.

- Provide special assistance for individuals with FASD within mainstream schools.

- Facilitate the creation of the special schools for learners with a learning disability (including individuals with FASD) that are not benefiting from mainstream schooling.

- Facilitate the education of parents on the needs and management of individuals with FASD.

Health-related management measures for FASD

The panel agreed that an FASD policy on the health-related management should contain strategies to

- Increase capacity building re diagnosis among health professionals.

- Facilitate FASD screening for all children that are known to have been prenatally exposed to alcohol.

- Provide diagnostic services for individuals.

- Promote diagnosis for school children, adolescents and adults to reduce rates of people who are left undiagnosed or misdiagnosed.
Table 4 A proposed guideline for policy on the prevention and management of FASD. (Continued)

- Promote appropriate referral pathways to services after diagnosis.

- Facilitate the creation of diagnostic centres in clinics, hospitals and communities.

- Facilitate the creation of national surveillance for FASD via reports from health professionals.

- Encourage routine consideration of FASD re the diagnosis and management of mental illness and developmental disorders.

- Provide integrated medical services for individuals with FASD.

Community/social-related management measures for FASD

The panel agreed that an FASD policy on the community-related management should contain strategies to

- Provide skills training and empowerment programmes for those who need it among individuals with FASD.

- Facilitate appropriate employment opportunities for individuals with FASD.

- Facilitate the training of community health workers/community-based workers/ youth care workers/ social workers and professionals within the judiciary system re FASD management.

- Improve the training of the biological and foster parents/caregivers regarding the management of FASD.

- Promote the empowerment of the parents/caregivers of individuals with FASD in the community.

- Promote the establishment of support systems for biological and foster parents/caregivers and individuals with FASD in the community.

- Promote the referral of parents and individuals with FASD to appropriate services.

- Provide effective counselling services for parents and individuals with FASD.

- Encourage family/community support for individuals with FASD.

- Provide support for individuals with FASD in child protection/foster care and the criminal justice system.

- Facilitate the creation of structure and supportive environment at home, school and beyond.

- Facilitate the provision of adequate information about individuals with for the adoptive parents.

\section{Discussion}

In this study, we aimed to develop a guideline that could assist policymakers in designing a holistic, comprehensive and multi-sectoral policy toward the prevention and management of FASD in South Africa. We considered a guideline as a document that contains evidence-based recommendations for the prevention and management of FASD and systematically developed statements capable of guiding policymakers to develop a holistic policy. This guideline has the potential to assist South African 
policymakers in developing a policy that will address FASD considering the acceptable consensus (85\%). Furthermore, it can be adapted or adopted to guide the development of policy for the prevention and management of FASD in other countries, especially within sub-Sahara Africa.

Our findings indicated good support for the proposed approaches and principles of FASD policy. This support confirms our proposition that policies designed to guide the prevention and management of FASD should be holistic, user- and caregiver-focused, culturally diverse and sensitive, considerate of needs across the lifespan, collaborative, have clear referral pathways, evidence-based and inter-departmental. These policies should also use the public health framework, and be human rights-based, inter-departmental, collaborative, culturally diverse and sensitive, evidence-based, cost-effective and family-centred approach [34, 36, 37]. If these perspectives are taken into consideration, the chances of obtaining a policy that enhances effective and sustainable programmes to prevent and manage FASD such as those developed in Australia and Canada are high [34, 36, 37].

While designing the initial guideline, based on the finding from previous phases, we considered adding that FASD policies should be women-centred. Nevertheless, the experts in the Delphi approach suggested that women-centeredness as a guiding principle has the tendency to promote stigmatisation and victim-blaming of women. Stigma may also influence the prevention and management of FASD as has been reported [9]. The opinions expressed by these participants are consistent with the current policy discourses to re-contextualise and decolonise FASD policies and reframe the problem of FASD in a way that avoids victim-blaming [33]. This principle supports the argument that drinking during pregnancy should not be criminalised [39]. Rather, women with alcohol problems should be assisted. Therefore, an FASD policy must address multiple factors (local and systemic) that predispose women to alcohol abuse [33]. An FASD policy should also address the social, structural and economic factors affecting health behaviour and examine the growing gap in health inequities [33]. Thus, the notion of having a policy which addresses upstream drivers (social determinants of health contributing to FASD) is in alignment with these arguments [40].

A FASD prevention policy should support the training of teachers for FASD prevention and awareness as well as classroom management and the modification of curriculum for the benefit of individuals with FASD in an educational setting. These findings are supported by studies conducted to examine the relational experience of educators and the education needs of children with FASD [41, 42]. The authors of these studies recommend specialised training for educators, empowering them to assist individuals with FASD in the classroom to maximise their potential $[41,42]$. Adequately trained educators can address the challenges of FASD in the classroom [43]. Goal 2 and priority 3 of the Canadian framework for action on FASD and the Australian national strategy for FASD, respectively emphasised on capacity development [36, 37]. We also recorded strong agreement on special assistance for individuals with FASD within the mainstream schools, so that they can benefit from inclusive education. Authors have advocated for specialised FASD classrooms and inclusive classrooms with FASD support [10].

Increasing the education and raising awareness around FASD, especially on the dangers of consuming alcohol during pregnancy in educational settings, clinics, communities and public places should also form a part of an FASD prevention policy. The need for education and awareness programmes is accentuated as research supports the efficacy of awareness intervention, particularly in areas where they are low [26]. These findings echo the urgent call for awareness in South Africa on the dangers of prenatal alcohol exposure and the overwhelming consequence of FASD on the lives of children, families and communities [3]. Comprehensive awareness and health promotion efforts correspond to the first level of prevention of the four-part model of prevention for FASD [44]. Awareness is also a core part of FASD policy strategies in Australia and Canada [34, 36, 37]. Therefore, awareness should be an integral part of a policy guiding the prevention and management of FASD [23].

The first step toward managing FASD in a proposed policy should include the screening of all children who are known to have been prenatally exposed to alcohol. This targeted screening has also been supported by another study [45]. The respondents, nevertheless, cautioned that it is dangerous to screen and diagnose without adequate care and management services. We also found a high agreement with the idea that an FASD policy should include programmes for screening for alcohol use at clinics and hospitals and proper documentation of the history of alcohol use. Therefore, healthcare providers must be equipped with the necessary skills to have informed discussions on alcohol use with women $[44,46]$. Discussing alcohol consumption and documenting its history also forms part of the recommendations of the Canadian framework for action on FASD, and the Australian action plans for FASD [34, 36, 37].

Training professionals (health care, social service, criminal justice and judiciary) regarding the prevention, screening, identification, diagnosis and management of FASD should also be considered in FASD policy. The continuous education and training of the professionals to improve their knowledge regarding FASD have also been advocated by some authors [47-51]. The 
training of these professionals is important because the prevention and management of FASD require a highly skilled multidisciplinary team [12-15]. Training is also essential as it is difficult to manage FASD because of its negative educational, health and social outcomes [52]. The training of parents and caregivers on the needs and how to care for individual with FASD should also be considered when designing policies to inform FASD programmes and interventions. Therefore, training is important, as the needs of individuals with FASD are enormous and parents/caregivers may not have sufficient knowledge of raising individuals with FASD [53]. Policies for FASD in other parts of the world have also considered the training of professionals in addressing FASD [34, 36, 37].

Our findings indicated the inclusion of support to the parents, caregivers, and individuals with FASD in policy which is relevant. These findings reflect one of the practice points for primary healthcare providers [PHCP], stipulating that "PHCP should be aware of FASD support services in their community and refer families to educational and family supports early" [54]. These findings also correspond to level four of the four-part model for FASD - postpartum support for new mothers and supports for child assessments and development [44]. The need for more support for families raising children with FASD has been indicated in a study [53]. Furthermore, Kapasi [55] found caregivers of individuals with FASD to have challenges, indicating the need for support. Challenges such as extra responsibility, difficulty in keeping a daily routine, feeling stigmatised and isolated, managing a child with antisocial behaviour problems and working with a child with diminished executive functioning [55]. Moreover, policies developed in Australia and Canada also advocated for support of the parents, caregivers, and individuals with FASD and community $[34,36,37]$.

\section{Strengths and limitations}

We followed a systematic empirical process to elicit information toward formulating the questionnaire. We developed the questionnaire using the findings of four different studies, with the aim of enhancing its content and quality. This process allowed us to gather credible information to be included in the questionnaire as all the participants had expertise/knowledge regarding FASD.

The questionnaire was also shared with local and international experts on FASD for comments and improvement through a discursive process to ensure the essential parts were covered before it was used for the Delphi approach. The use of the Delphi approach provides the opportunity for participants to provide their opinions without fear of having differing views with others. This approach led to a wide range of opinions, which improved the guideline. The Delphi approach provides a strong basis for the construct validity of the questionnaire with participants being able to validate their initial responses and identify areas of uncertainty. The Delphi approach used in this study also allows controlled feedback, which provided the researchers with an opportunity to organise information, if applicable remove duplicates before exchanging the information with the experts.

The purposive and snowballing sampling method used may not have provided the true representation of all the individuals who are knowledgeable or working in the area of FASD. Therefore, this method could have limited the conclusion drawn from the study. A lack of participation from criminal justice and judicial professionals is also a limitation of this study, as their perspectives have been considered essential in the literature. Additionally, we did not include individuals with FASD and their biological or foster parents and caregivers.

\section{Conclusion}

FASD in South Africa deserves urgent attention, especially from the government in terms of the policy to coordinate relevant prevention and management programmes and interventions. Developing a comprehensive and inter-sectoral policy to guide programmes and interventions for the prevention and management of FASD could be a good starting point. The guideline developed has the potential to assist the policymakers in the development of a holistic, multisectoral and comprehensive policy for FASD or could streamline discussions on an FASD policy in other relevant contexts.

\section{Supplementary information}

Supplementary information accompanies this paper at https://doi.org/10. 1186/s12913-019-4677-x

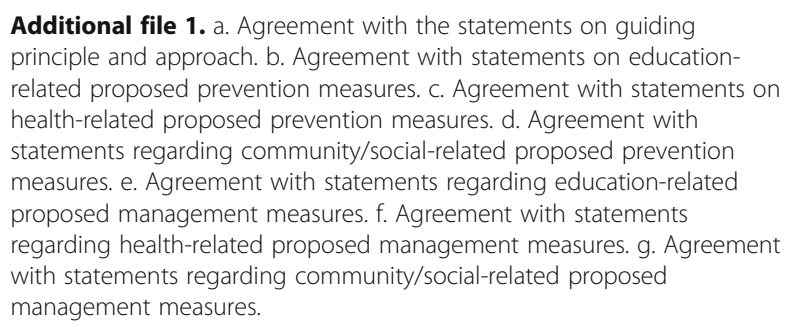

\section{Abbreviation}

ARBD: Alcohol-related birth defects; ARND: Alcohol-related

neurodevelopmental disorder; FARR: Foundation for alcohol related research;

FAS: Fetal alcohol syndrome; FASD: Fetal alcohol spectrum disorder;

NPO: Non-profit Organisation; PFAS: Partial fetal alcohol syndrome;

PHCP: Primary healthcare providers; SPSS: Statistical Package for Social

Science; WHO: World Health Organization 


\section{Acknowledgements}

We thank all the experts who participated in the study (Carol Bower, Hayley Passmore, Leana Olivier, Leslie London, Lizahn Cloete, Martyn Symons, Natasha Reid and Rochelle Watkins).

Also, we would like to thank the following organisations: Abedare Primary School, Bel Porto School, FASfacts, Home of Hope, Foundation for Alcohol Related Research, Karitas Schools, Khayelitsha (Site B) Community Health Centre, Khayelitsha Hospital, Mowbray Maternity Hospital, Red Cross War Memorial Children's Hospital, Western Cape Department of Education, Western Cape Department of Health, Western Cape Department of Social Development and Western Cape Substance Abuse Forum. In addition, we would like to thank the peer reviwers (Debra Jackson and Roy Grant) for their constructive comments.

\section{Authors' contributions}

The study was conceived and conceptualised by BOA and FCM. BOA, FCM and $A M B$ contributed to the development of the methodology of this study. $\mathrm{BOA}$ and FCM analysed and interpreted the data. BOA wrote the first draft of the manuscript. FCM and AMB provided editorial and content input to improve the manuscript. All the authors read and approved the final manuscript.

\section{Funding}

No funding declared.

\section{Availability of data and materials}

More information on data from this study is available by contacting the corresponding author

\section{Ethics approval and consent to participate}

This study is a part of the larger study. The approval for the larger study was obtained from the research ethics committee of the University of the Western Cape, Cape Town, South Africa. (BM/16/4/4). A written consent was obtained from all the participants.

\section{Consent for publication}

Not applicable.

\section{Competing interests}

The authors declare that they have no competing interests.

\section{Author details}

'School of Public Health, University of the Western Cape, Cape Town 8001, South Africa. ${ }^{2}$ Department of Social Work, University of the Western Cape, Cape Town, South Africa.

\section{Received: 12 June 2019 Accepted: 24 October 2019}

Published online: 06 November 2019

\section{References}

1. Lange S, Probst C, Gmel G, et al. Global prevalence of fetal alcohol Spectrum disorder among children and youth. JAMA Pediatr. 2017;171:948

2. Home - FARR SA, https://www.farrsa.org.za/ (Accessed 7 Jan 2019).

3. Olivier L, Curfs $L M G$, Viljoen DL. Fetal alcohol spectrum disorders: prevalence rates in South Africa. South African Med J. 2016;106:103-6.

4. May P, De Vries M, Marais A-S, et al. Replication of high fetal alcohol spectrum disorders prevalence rates, child characteristics, and maternal risk factors in a second sample of rural communities in South Africa. Int J Environ Res Public Health. 2017:14:522.

5. Urban MF, Olivier $L$, Viljoen $D$, et al. Prevalence of fetal alcohol syndrome in a south African city with a predominantly black African population. Alcohol Clin Exp Res. 2015:39:1016-26.

6. Denny LC, S Blitz R. Fetal alcohol syndrome and fetal alcohol Spectrum disorders. Am Fam Physician. 2017:96:515-22.

7. Williams JF, Smith VC, ABUSE COS. Fetal alcohol Spectrum disorders. Pediatrics. 2015:136:e1395-406.

8. Badry D, Choate P. Social work \&amp; social sciences review. Whiting \& Birch Ltd, 2015. https://journals.whitingbirch.net/index.php/SWSSR/article/view/ 795/867. Accessed 7 Jan 2019.
9. Bell E, Andrew G, Di Pietro N, et al. It's a shame! Stigma against fetal alcohol Spectrum disorder: examining the ethical implications for public health practices and policies. Public Health Ethics. 2016;9:65-77.

10. Millar JA, Thompson J, Schwab D, et al. Educating students with FASD: linking policy, research and practice. J Res Spec Educ Needs. 2017;17:3-17.

11. Select Committee on Action to Prevent Foetal Alcohol Spectrum Disorder. The preventable disability. 2015. https://parliament.nt.gov.au/_data/assets/ pdf_file/0005/363254/Final_FASD_Report.pdf. Accessed 1 Nov 2019.

12. Chasnoff IJ, Wells AM, King L. Misdiagnosis and missed diagnoses in Foster and Adopted children with prenatal alcohol exposure. Pediatrics. 2015;135:264-70.

13. Burd L. FASD and ADHD: are they related and how? BMC Psychiatry. 2016;16:325.

14. Benz J, GA CR. History, challenges, and future directions. 2009;14:231-7.

15. Jamieson PA. The challenge of supporting children with fetal alcohol Spectrum disorder in Aotearoa New Zealand : a narrative literature review; 2017.

16. Reid N, Dawe S, Shelton D, et al. Systematic review of fetal alcohol spectrum disorder interventions across the life span. Alcohol Clin Exp Res. 2015:39:2283-95.

17. Chudley AE, Conry J, Cook JL, et al. Fetal alcohol Spectrum disorder: Canadian guidelines for diagnosis. CMAJ. 2005;172:S1-S21.

18. Cook JL, Green CR, Lilley CM, et al. Fetal alcohol spectrum disorder: a guideline for diagnosis across the lifespan. CMAJ. 2016;188:191-7.

19. Hoyme HE, Kalberg WO, Elliott AJ, et al. Updated clinical guidelines for diagnosing fetal alcohol spectrum disorders. Pediatrics. 2016;138:e20154256.

20. Jonsson E, Salmon A, Warren KR. The international charter on prevention of fetal alcohol spectrum disorder. Lancet Glob Health. 2014;2:e135-7.

21. Rendall-Mkosi K, London L, Adnams C, et al. Fetal alcohol spectrum disorder in South Africa: situational and gap analysis. Pretoria: UNICEF 2008. https://www.unicef.org/southafrica/SAF_resources_fetalalcohol.pdf (Accessed 14 Sept 2017)

22. Roozen S, Black D, Peters G-JY, et al. Fetal alcohol Spectrum disorders (FASD): an approach to effective prevention. Curr Dev Disord Rep. 2016;3:229-34.

23. Becoming RD, FASD. Informed: strengthening practice and programs working with women with FASD. Subst Abus. 2016;10:13-20.

24. Adebiyi BO, Mukumbang FC, Cloete LG, et al. Policymakers' perspectives towards developing a guideline to inform policy on fetal alcohol Spectrum disorder: a qualitative study. Int J Environ Res Public Health. 2019;16(6):945. https://doi.org/10.3390/ijerph16060945 Epub ahead of print.

25. Adebiyi BO, Mukumbang FC, Cloete LG, et al. Exploring service providers' perspectives on the prevention and management of fetal alcohol spectrum disorders in South Africa: a qualitative study. BMC Public Health. 2018;18(1): 1238. https://doi.org/10.1186/s12889-018-6126-x Epub ahead of print.

26. Chersich MF, Urban M, Olivier $L$, et al. Universal prevention is associated with lower prevalence of fetal alcohol spectrum disorders in northern cape, South Africa: a multicentre before-after study. Alcohol Alcohol. 2012:47:67-74.

27. May PA, Marais A-S, Gossage JP, et al. Case management reduces drinking during pregnancy among high risk women. Int J Alcohol Drug Res. 2013;2:61-70.

28. Adebiyi BO, Mukumbang FC, Beytell A-M. To what extent is fetal alcoho Spectrum disorder considered in policy-related documents in South Africa? A document review. Heal Res Policy Syst. 2019;17:46.

29. London leslie. South Africa fails to tackle its high foetal alcohol syndrome rate; 2015. http://theconversation.com/south-africa-fails-to-tackle-its-highfoetal-alcohol-syndrome-rate-46791 (Accessed 18 Dec 2018)

30. Adebiyi BO, Mukumbang FC, Okop KJ, et al. A modified Delphi study towards developing a guideline to inform policy on fetal alcohol spectrum disorders in South Africa: a study protocol. BMJ Open. 2018;8:e019907.

31. Foundation for Alcohol Related Research (FARR). What we do - FARR SA; 2018. https://www.farrsa.org.za/what-we-do/ (Accessed 16 July 2018)

32. FASfacts. FASfacts runs FAS prevention campaigns in schools and communities; 2018. http://www.fasfacts.org.za/About-Us/FASfactsProgrammes (accessed 16 July 2018)

33. Hunting G, Browne AJ. Decolonizing policy discourse: reframing the 'problem' of fetal alcohol spectrum disorder. Women's Heal Urban Life. 2012;11:35-53

34. Foundation for Alcohol Education and Research. The Australian fetal alcohol Spectrum disorders action plan 2013-2016; 2013. https://www.fare.org.au/ wp-content/uploads/research/FARE-FASD-Plan.pdf (Accessed 5 Feb 2018) 
35. Adnams CM. Fetal alcohol spectrum disorder in Africa. Curr Opin Psychiatry. 2017;30:108-12.

36. Public Health Agency of Canada. Fetal alcohol spectrum disorder (FASD) : a framework for action. Health Canada; 2003. https://www.canada.ca/en/ public-health/services/reports-publications/fetal-alcohol-spectrum-disorderfasd-framework-action.html (Accessed 8 Aug 2018)

37. Australian Government. Title: National Fetal Alcohol Spectrum Disorder Strategic Action Plan; 2018. http://www.dpmc.gov.au/government/ commonwealth-coat-arms (Accessed 22 Feb 2019)

38. Adebiyi BO, Mukumbang FC, Erasmus C. The distribution of available prevention and management interventions for fetal alcohol Spectrum disorder (2007 to 2017): implications for collaborative actions. Int J Environ Res Public Health. 2019;16:2244.

39. Gardner J. South African journal of bioethics and law. S Afr J Bioeth Law. 2016;9:26.

40. Sanders J, Currie CL. Looking further upstream to prevent fetal alcohol spectrum disorder in Canada. Can J Public Heal. 2014;105:e450-2.

41. Van Schalkwyk I, Marais S. Educators' relational experiences with learners identified with fetal alcohol spectrum disorder. S Afr J Educ. 2017;37:1-9.

42. Millians MN. Educational needs and Care of Children with FASD. Curr Dev Disord Rep. 2015;2:210-8.

43. Blackburn C, Whitehurst T. Fetal alcohol spectrum disorders (FASD): raising awareness in early years settings. Br J Spec Educ. 2010;37:122-9.

44. Poole N, Schmidt RA, Green C, et al. Prevention of fetal alcohol spectrum disorder: current Canadian efforts and analysis of gaps. Subst Abus. 2016;10:1-11.

45. Watkins RE, Elliott EJ, Wilkins $A$, et al. Recommendations from a consensus development workshop on the diagnosis of fetal alcohol spectrum disorders in Australia. BMC Pediatr. 2013;13:156.

46. Coons KD, Watson SL, Yantzi NM, et al. Health care students' attitudes about alcohol consumption during pregnancy: responses to narrative vignettes. Glob Qual Nurs Res. 2017:4:2333393617740463.

47. Payne J, France K, Henley N, et al. Changes in health professionals' knowledge, attitudes and practice following provision of educational resources about prevention of prenatal alcohol exposure and fetal alcohol Spectrum disorder. Paediatr Perinat Epidemiol. 2011;25:316-27.

48. Payne JM, Watkins RE, Jones HM, et al. Midwives' knowledge, attitudes and practice about alcohol exposure and the risk of fetal alcohol spectrum disorder. BMC Pregnancy Childbirth. 2014;14:377.

49. Mutch RC, Jones HM, Bower C, et al. Fetal alcohol Spectrum disorders: using knowledge, attitudes and practice of justice professionals to support their educational needs. J Popul Ther Clin Pharmacol. 2016;23:e77-89.

50. Passmore HM, Mutch RC, Burns S, et al. Fetal alcohol Spectrum disorder (FASD): knowledge, attitudes, experiences and practices of the Western Australian youth custodial workforce. Int J Law Psychiatry. 2018:59:44-52.

51. Cox LV, Clairmont D, Cox S. Knowledge and attitudes of criminal justice professionals in relation to fetal alcohol Spectrum disorder. Can J Clin Pharmacol. 2008;15:e306-13.

52. Centers for Disease Control and Prevention (CDC). Fetal alcohol Spectrum disorders (FASDs) | NCBDDD | CDC; 2018. https://www.cdc.gov/ncbddd/ fasd/index.html (Accessed 7 May 2018)

53. Coons KD, Watson SL, Schinke RJ, et al. Adaptation in families raising children with fetal alcohol spectrum disorder. Part I: what has helped. J Intellect Develop Disabil. 2016;41:150-65.

54. Hanlon-Dearman A, Green CR, Andrew G, et al. Anticipatory guidance for children and adolescents with fetal alcohol spectrum disorder (FASD): practice points for primary health care providers, 2015. https://www. researchgate.net/profile/Ana_Hanlon-Dearman/publication/271140236_ Anticipatory_Guidance_for_Children_and_Adolescents_with_Fetal_Alcohol_ Spectrum_Disorder_FASD_Practice_Points_for_Primary_Health_Care_ Providers/links/5504684b0cf2d60c0e677bc9/Ant (Accessed 7 June 2017).

55. Kapasi A. 'Caregivers" "Experiences Raising a Child with Fetal Alcohol Spectrum Disorder"'. Other psychology commons; 2015. https://irlib.uwo.ca/ etd/2713 (Accessed 22 Feb 2019)

\section{Publisher's Note}

Springer Nature remains neutral with regard to jurisdictional claims in published maps and institutional affiliations.

Ready to submit your research? Choose BMC and benefit from:

- fast, convenient online submission

- thorough peer review by experienced researchers in your field

- rapid publication on acceptance

- support for research data, including large and complex data types

- gold Open Access which fosters wider collaboration and increased citations

- maximum visibility for your research: over $100 \mathrm{M}$ website views per year

At BMC, research is always in progress.

Learn more biomedcentral.com/submissions 